Décadrages Décadrages

cinéma, à travers champs Cinéma, à travers champs

$6 \mid 2005$

Train et cinéma

\title{
La perception ferroviaire
}

\section{Livio Belloï}

\section{OpenEdition}

\section{Journals}

Édition électronique

URL : https://journals.openedition.org/decadrages/494

DOI : $10.4000 /$ decadrages.494

ISSN : 2297-5977

\section{Éditeur}

Association Décadrages

\section{Édition imprimée}

Date de publication : 10 octobre 2005

Pagination : 70-83

ISBN : 978-2-9700582-2-9

ISSN : 2235-7823

Référence électronique

Livio Belloï, « La perception ferroviaire », Décadrages [En ligne], 6 | 2005, mis en ligne le 21 janvier 2014, consulté le 27 mars 2022. URL : http://journals.openedition.org/decadrages/494 ; DOI : https:// doi.org/10.4000/decadrages.494

(B) Décadrages 


\section{La perception ferroviaire}

\section{par Livio Belloï}

1 Le titre est de la rédaction. Nous reproduisons deux extraits du livre en question, pp. 100-107 et pp. 196-207.

2 Le terme de "vue", qu'on trouve notamment dans les catalogues des premiers producteurs de films, s'est d'abord appliqué à des films "uniponctuels", en ce sens qu'ils ne comportent qu'un seul plan, pris depuis un point de vue unique.

3 Rappelons à ce propos que Belloï est l'auteur d'un important article consacré à l'"archéologie" des genres cinématographiques, c'est-àdire aux conditions de possibilité de leur émergence progressive, aux débuts du cinéma, dans les discours tenus sur les films au niveau aussi bien des instances de production que des ins tances de réception: "Répétitions, variations, reconfigurations: à propos du concept de 'genre dans le cinéma des premiers temps", dans L. Quaresima, A. Rengo, L. Vici (éd.), La nascita dei generi cinematographici, Udine, Forum, 1999, pp. 69-86.
Chercheur qualifié du Fonds National de la Recherche Scientifique (Belgique), attaché à l'Université de Liège, Livio Belloï est l'auteur du Regard retourné. Aspects du cinéma des premiers temps (Québec-Paris, Nota Bene-Méridiens Klincksieck, 2001), ouvrage remarquable et trop peu remarqué, dont la disponibilité en librairie s'avère parfois problématique, en raison de divers aléas éditoriaux. C'est pourquoi nous tenons à reproduire ci-dessous, avec l'accord de l'auteur, deux passages de son livre qui abordent frontalement la problématique de notre dossier 1 .

Le premier passage, "Le train comme projectile: logique de la vue ferroviaire», est extrait du chapitre II (le livre en comporte quatre), intitulé «La fiction de la vue attentatoire»2. Ce chapitre est consacréa une des modalités de cette «confrontation exbibitionniste» entre le film et son spectateur dont Tom Gunning a pu faire un des traits définitoires du mode de représentation du cinéma des premiers temps (en gros jusque vers 1908). La «vue attentatoire» aurait comme particularité non seulement de retourner son regard au spectateur, mais surtout de pouvoir emprunter d'autres voies que celle du seul regard-caméra. C'est dans ce cadre que Bellö̈ porte son attention sur ce qu'on peut considérer comme deux sous-genres ${ }^{3}$ de la «vue de plein air», la vue marine et la vue ferroviaire. Toutes deux figurent un mouvement (naturel dans le premier cas, mécanique dans le second) qui peut s'exercer en direction du spectateur et par là même faire mine de mettre en crise cette "ségrégation des espaces perceptifs» (espaces écranique et proprioceptif) dont a parlé la filmologie.

Le second passage, "La perception ferroviaire: de la dispersion à la synthèse», est extrait du chapitre III, "La fiction de la vue panoramique». Ce chapitre réinterroge l'inscription de ce type de vue dans l'histoire de la représentation panoramique (picturale/photographique/cinématographique) pour mettre en évidence ce qu'a de singulier la condition d'un spectateur de cinéma à la fois immobilisé (contrairement à ce qu'il en est généralement dans le cas $d u$ panorama pictural ou photographique, où la mobilité du spectateur est une des conditions $d u$ «fonctionnement» $d u$ dispositif) et confronté à un mouvement généralisé, mouvement dans le plan et mouvement du plan, lorsque l'appareil de prise de vue est disposé dans un mobile tel qu'un train. (ac) 
Pour être bien connue et bien attestée aujourd'hui, la rencontre originaire entre ces deux emblèmes de la modernité que sont le train et le cinéma garde quelque chose de fascinant et d'énigmatique à la fois, tant elle semble inaugurer un réseau de correspondances suivies, un véritable système conjugué de relations croisées et singulièrement durables. Au point que, porté par un désir somme toute très légitime, l'on peut parfois se trouver tenté d'entreprendre une histoire générale du cinéma à la lumière du train, sous son seul jour 4 .

En regard du cinéma des premières années, on le sait, le train ne se sera pas contenté de composer un horizon, de fonder un véritable motif obsessionnel, de L'arrivée d'un train en gare de La Ciotat (Lumière, $\mathrm{n}^{\circ}$ 653) à The Girl and her Trust (D. W. Griffith, Biograph, 1912), en passant, bien sûr, par The Great Train Robbery (E. S. Porter, Edison, 1903) : il aura également - plus sûrement, plus fondamentalement peut-être - informé en profondeur ses procédures de montage et précipité, dans son sillage, l'éclosion de schémas narratifs élémentaires, parmi lesquels doit évidemment figurer, à la place qui lui revient de droit, celui de l'alternance $\mathbf{5}$. Et lorsque le train n'offrit pas au premier cinéma un motif visuel ou un argument narratif, il se mit tout simplement au service de son dispositif d'exhibition, tantôt comme environnement de projection (le fameux dispositif Hale's Tours and Scenes of The World, développé aux Etats-Unis à partir de 1904-1905), tantôt à titre de générateur, de véritable dynamo (telle la «locomobile» dans certaines exploitations foraines) ${ }^{6}$.

Il est bien question, à tous égards, d'une affinité élective, nouée, entrelacée dans les rapports que les deux machines entretiennent au mouvement et au visible. Jonathan Crary a bien mis en relief la question fondamentale que le défilement ferroviaire adresse à la perception, la manière dont il inquiète et interroge le regard. Crary évoque notamment les travaux du mathématicien anglais Peter Mark Roget qui, dès 1825 , publie un compte rendu relatif aux diverses illusions d'optique produites par les roues d'un train en mouvement lorsque celles-ci sont aperçues au travers des barres verticales d'une palissade. Selon Roget, deux types d'illusion adviennent en pareille circonstance : soit les rayons s'offrent au regard comme immobiles (annulation du mouvement), soit ils paraissent tourner à rebours (réversion). Ces observations suggèrent à Roget l'importance de l'emplacement investi par l'observateur (ici, face à un objet-écran qui fait interposition), eu égard au phénomène de persistance des images rétiniennes (Faraday et sa roue, Plateau et son phénakistiscope s'engageront sur une voie analogue) ${ }^{7}$.
4 C'est d'ailleurs à semblable projet que Lynne Kirby a voulu s'atteler, à l'échelle du seul cinéma muet. Voir Parallel Tracks. The Railroad and Silent Cinema, Duke University Press, 1997.

5 Sur ce point, voir l'important article d'André Gaudreault, "De L'arrivée d'un train à The Lonedale Operator: une trajectoire à parcourir ", dans Jean Mottet (éd.), David Wark Griffith, L'Harmattan, Paris, 1984, pp. 44-71.

6 Sur le Hale's Tour et les dispositifs apparentés, voir l'article fondamental de Raymond Fielding, "Hale's Tours: Ultrarealism in the Pre-1910 Motion Picture ", dans John L. Fell (éd.), Film Before Griffith, University of California Press, Berkeley/Los Angeles, 1983, pp. 116-130. Sur le même point, voir encore Charles Musser, "The Travel Genre in 1903-1904: Moving Toward Fictional Narrative ", in Iris, vol. 2, $\mathrm{n}^{\circ} 1$, 1984, pp. 47-59, ainsi que quelques remarques - éparses mais néanmoins importantes dans The Emergence of Cinema. The American Screen to 1907, Charles Scribner's Sons, New York, 1990; voir également Noël Burch, La lucarne de l'infini, Nathan, Paris, 1991 [1990], pp. 38-42. Quant à la "locomobile", elle constitue un cas tout à fait singulier d'attelage entre les deux machines: un attelage qui ne concerne pas la prise de vue (comme tel sera le cas pour le panorama ferroviaire, sur lequel nous reviendrons dans le cadre d'une section ultérieure), mais exclusivement la projection. A cet égard, on se reportera à la description de ce dispositif donnée par Jacques Deslandes et Jacques Richard (Histoire comparée du cinéma, t. II: du cinématographe au cinéma 1896-1906, Casterman, Tournai, 1968, p. 196): "Actionnant une dynamo [la locomobile] brûlait du coke et ressemblait fort à ces batteuses qui fonctionnaient encore dans certains villages de France il y a une trentaine d'années. Mais elle était ornée de cuivres étincelants et rigoureusement silencieuse. Cette espèce de locomotive à cheminée très haute d'où s'échappent de gros nuages blancs constitue bien souvent, dans les premières années du siècle, le clou de la parade des cinémas forains. "Nouvel indice de ce que, d'entrée de jeu, le train et le cinéma vont littéralement de pair.

7 Voir Jonathan Crary, L'art de l'observateur, Jaqueline Chambon, Nîmes, 1994 [1990], pp. 153-155. 
8 A ce sujet, voir Marc Baroli, Le train dans la littérature française, Editions de l'Ecole technique d'imprimerie "Notre famille", Paris, 1963; et Jacques Noiray, Le romancier et la machine, t. I: L'univers de Zola, Corti, Paris, 1981.

9 Wolfgang Schivelbusch, Histoire des voyages en train [1977], Le Promeneur/Quai Voltaire, Paris, 1990, p. 133.

10 Sur ce point, voir notamment l'étonnant texte de l'économiste saint-simonien Constantin Pecqueur (1839), cité dans Wolfgang Schivelbusch, Histoire des voyages en train, op. cit., pp. 75-76.
Objet mouvant et visible, énigme posée à la perception, le train a très tôt cristallisé un imaginaire très riche et très chargé bien avant que le cinéma ne s'en empare et ne le consacre en objet de prédilection. Mieux encore, la machine-train aura fixé sur elle, à l'état de condensé, l'ambivalence profonde et intrinsèque dont toutes les machines sont investies dans l'imaginaire de la révolution industrielle: ambivalence dont témoignera exemplairement et à l'envi le roman ferroviaire (en pleine efflorescence dans les années 1870-1890) ${ }^{8}$, et dont les premiers films de train sauront assurément perpétuer la trace et prolonger les effets.

Selon Wolfgang Schivelbusch, «la perception primitive du chemin de fer se caractérise par une ambivalence remarquable», qui place la machine au centre de réseaux complexes et clivés, en oscillation perpétuelle entre sa face claire et sa face sombre, sa part rationnelle et sa part fantasmatique ${ }^{9}$. Dans sa dimension rationnelle, la machine se donne avant tout comme l'emblème le plus visible de la révolution industrielle : produit d'un asservissement des nouvelles forces motrices et d'une rationalisation de la nature, elle s'expose comme la consécration objective d'une idéologie résolument progressiste et trouve rapidement à s'instaurer, aux yeux de certains, en véritable instrument d'émancipation pour l'Humanité10. Dans cette perspective unanimiste et égalitariste, l'homme passe pour dominer unilatéralement la machine, pour exercer, à son endroit, une emprise stricte et infaillible. Et, si rapprochement spatial rime volontiers, en l'espèce, avec rapprochement social, il ne faut par ailleurs y voir que l'affirmation d'une volonté de domination étendue à un univers désormais offert à tous les parcours et à tous les quadrillages.

Sous son aspect fantasmatique, la machine apparaît, au rebours, comme une entité dominante et non plus dominée. C’est sans doute sous cet éclairage qu'il convient de lire, dans le roman ferroviaire français des années 1870-1890, la figuration récurrente de la machine sous les aspects d'un être inquiétant, animé d'une vie propre, soumis à des affections, à des caprices, voire à des colères. On sait le traitement exemplaire que Zola réservera à ce motif dans La bête humaine. On sait également tout le parti qu'Abel Gance voudra en tirer avec La roue (1921-1923): entre la Lison de Jacques Lantier et la Norma-Compound de Sisif, bien des points de convergence se laissent repérer, qui spécifient de concert tous les termes d'un rapport complexe, tortueux, presque passionnel de l'humain au machinique (et réciproquement). Dans ce cas d'espèce - et en dépit des apparences -, la relation de l'homme à la machine est vécue sur un mode profondément dysphorique et anxiogène. C'est qu'advient toujours le moment fatidique où "l'homme ne se reconnaît plus dans 
les objets qu'il a créés »11. Conjoignant dans le même dessin la double série métaphorique du bouillonnement diabolique et de l'animalité monstrueuse, la machine passe alors pour échapper à son créateur et pour engager avec lui une lutte inégale et sans merci (sous cet angle, la machine rejoue d'ailleurs assez fidèlement, on le sait, l'un des aspects du mythe frankensteinien).

Quant au roman ferroviaire, Jacques Noiray a justement souligné que ce retournement convoque le plus souvent deux figurations récurrentes et complémentaires: d'une part, le train comme machine aveugle, laquelle, dans sa logique, dans son inexorable rectitude, se propulse dans l'espace suivant une direction immuable, indifférente à toute forme d'obstacle, fût-il humain (aveugle, la machine sera alors perçue ni plus ni moins que comme un projectile, le voyage lui-même s'éprouvant comme un tir à travers le paysage 12). Corollairement, le train se représentera volontiers sous les traits d'une machine meurtrière, écrasant, balayant tout sur son passage, de son inéluctable mouvement13.

C'est dans l'accident de chemin de fer que s'opérera, exemplairement et dramatiquement, la conjonction des deux traits. Dessinant les contours d'un vaste paysage névrotique, la collision ferroviaire fera l'objet, dès les années 1860, d'une abondante littérature scientifique, où se feront jour les préoccupations les plus diverses. Selon Schivelbusch, ce sont ces débats - souvent vifs et contradictoires - qui mèneront, de proche en proche, à l'élaboration de la théorie freudienne du pare-excitation et de sa rupture, en passant par les explications physiologiques de John Eric Erichsen et leur réfutation en règle par Herbert W. Page ${ }^{\mathbf{1 4}}$.

Machine visible, le train se sera donc également imposé en machine épistémologique, propre à déclencher, à innerver sur son passage une pensée tantôt physiologique, tantôt psychologique. C'est que la machine était porteuse, par essence, d'une puissante charge traumatique et qu'en cela, elle ne pouvait que susciter les interrogations les plus diverses (celles, notamment, d'une "physiologie» ou d'une "psychologie des chemins de fer", comme s'intitulent nombre de traités publiés dans la seconde moitié du $\mathrm{XX}^{\mathrm{e}}$ siècle), et, plus encore, qu'alimenter de ses motifs tel sous-genre littéraire (le roman ferroviaire, sans doute la forme la plus pure de littérature de gare). L'on aperçoit donc clairement à quel point le motif ferroviaire était susceptible, par delà, d'intéresser les premières formes d'image-attraction. Car s'il est vrai que le voyage en chemin de fer peut s'éprouver comme un tir à travers le paysage, alors filmer le passage d'un train revient très exactement à filmer la course d'un projectile. En somme, toutes les conditions étaient réunies pour que se noue, entre le motif (ferroviaire) et la forme (attentatoire), une relation de captation
11 Jacques Noiray, Le romancier et la machine, op. cit., p. 425.

12 Wolfgang Schivelbusch, Histoire des voyages en train, op. cit., p. 59.

13 Jacques Noiray, ibid.

14 Sur ces points, voir Wolfgang Schivelbusch, Histoire des voyages en train, op. cit., pp. 137160 (pour le débat entre les approches physiologique et psychologique) et 161-171 (pour la formulation de la thèse freudienne). Dans un ouvrage daté de 1875, Erichsen donne une description très éclairante des propriétés spécifiques de l'accident de chemin de fer: "On doit se souvenir du caractère propre des accidents de chemin de fer: ils fondent sur la victime sans avertissement préalable, on dispose au mieux de quelques secondes pour s'y préparer, l'abandon total de l'homme au milieu de ces masses en mouvement rend ces accidents particulièrement effrayants. Dans la plupart des accidents ordinaires, par exemple une voiture dont le cheval s'emporte, la victime dispose de quelques minutes pour se préparer. Elle peut rassembler son énergie et faire une tentative pour se sauver; sa situation ne lui apparaît pas totalement désespérée. La force de la collision et le trouble qui s'ensuivent dans un accident de chemin de fer, les cris des blessés et peut-être la vue des victimes de la catastrophe suscitent une impression psychique considérablement plus profonde que lors des accidents ordinaires. "L'idée que l'accident fond littéralement sur la victime conforte la prégnance du train comme projectile. 
et de captivation réciproque. Le moins que l'on puisse dire, dès lors, c'est que le sillon était déjà tout tracé et qu'il n'était, en définitive, que de s'y engager.

Dans le dessein d'en explorer au mieux les diverses configurations, il s'agira ici d'aborder l'examen de trois vues ferroviaires contemporaines les unes des autres: l'une, illustre, presque mytique, L'arrivée d'un train en gare de La Ciotat (Lumière, $\mathrm{n}^{\circ} 653$ ); les deux autres, moins fréquemment évoquées et pourtant exemplaires: The Empire State Express (W. K. L. Dickson, Biograph, 1896) et The Black Diamond Express (Edison, 1896). En l'occurrence, il sera surtout question de voir en quoi la vue ferroviaire s'entend à décliner les principes de la vue attentatoire, et comment, simultanément, inséparablement, elle se fait le théâtre de cet «équilibre instable» (Schivelbusch), de cette ambivalence constitutive dans laquelle se tient la machine lancée en trombe sur la voie.

Entre L'arrivée d'un train en gare de La Ciotat, The Empire State Express et The Black Diamond Express, il y a plus qu'un motif à peu près identique et qu'une mise en cadre un peu analogue. En effet, ces trois vues sont prises en un réseau intertextuel particulièrement serré : le film Biograph s'inspirerait ouvertement du film Lumière, alors que le film Edison serait lui-même formé à l'imitation du film Biograph. Ces trois vues ferroviaires sont de toute évidence produites dans un contexte de très forte concurrence qui non seulement surdétermine une pratique plagiaire, typique de la "circulation des signes» (Burch) propre aux premiers temps du cinéma, mais qui doit encore, à n'en pas douter, informer en profondeur la recherche des effets attractionnels (outre l'imitation, la concurrence suppose toujours la surenchère).

Il semble que les lieux, les sites effectifs de prise de vue procurent déjà l'occasion d'un partage, d'une distribution paradigmatique. Sous cet angle, la vue Lumière se démarque nettement des deux autres, dans la mesure où elle opte pour un point d'arrivée, un point de butée (la gare, le quai), alors que les vues Biograph et Edison sélectionnent, quant à elles, un point de passage, une sorte de no man's land entre deux gares (pour l'une, un espace d'entre-deux relativement désertique, borné par des collines à l'arrière-plan ; pour l'autre, un paysage vallonné, à la végétation sauvage - avec des cheminots au travail à l'entour de la voie dans l'une comme dans l'autre). Il est d'ailleurs remarquable que le poste de prise de vue finalement arrêté par les opérateurs respectifs ait trouvé à se déterminer, d'emblée, suivant deux conceptions distinctes du réseau ferroviaire et de ses distances: alors que le réseau européen (celui au sein duquel Lumière officie) tend alors à se déployer sur des distances 
relativement courtes, scandées par de nombreux arrêts en gare, le réseau américain (celui-là même que les vues Biograph et Edison prennent en ligne de mire) se spécifie, pour sa part, en cela qu'il couvre des distances beaucoup plus longues et qu'il se dispense volontiers de tout point d'arrêt. Ainsi que Schivelbusch le fait observer, "[t]andis que le voyage en Europe inclut les gares et leurs installations comme le buffet, les toilettes, il n’y a, en Amérique, pour ainsi dire pas de gare»15. D’un côté, c'est donc la station qui se donne comme le lieu cardinal du réseau; de l'autre, c'est la voie qui s'en offre comme l'assise.

Cette distinction n'est évidemment pas sans incidence: du paradigme de l'arrivée (en gare) à celui du passage (entre deux gares), ce sont, d'emblée, deux rapports tout à fait distincts au mouvement machinique, deux paysages différents, deux sphères de la vie sociale (le loisir vs le travail) et deux classes de sujets relativement hétérogènes (les usagers vs les cheminots) qui viennent à se figurer dans le cadre. Au point que l'on serait dès à présent fondé à penser que, sous des effets de surface analogues, le motif de l'arrivée et celui du passage n'enferment pas tout à fait les mêmes questions.

\section{LA PERCEPTION FERROVIAIRE : DE LA DISPERSION À LA SYNTHÈSE}

Comme l'on sait, la pratique qui consistait à arrimer l'appareil de prise de vue sur un mobile quelconque, si elle trouva son lieu originaire et mythique sur la gondole de Promio, ne tarda cependant pas à s'en émanciper, pour se tourner résolument vers la locomotion ferroviaire, qui lui offrait un autre site, sans doute plus commode et plus productif. Quelques mois après que Dickson eut tourné The Empire State Express, la firme Biograph mit ainsi sur le marché Through the Haverstraw Tunnel, une vue panoramique tournée depuis l'avant d'une locomotive, et bien faite, en cela, pour enchérir sur les effets cultivés par la vue ferroviaire originaire.

On observera au passage que le dispositif même de la vue panoramique, sous son espèce ferroviaire, devait, en définitive, occuper un emplacement cardinal dans l'imagerie attachée, par convention, aux tout premiers temps du cinéma. Si L'arrivée d’un train en gare de La Ciotat a depuis longtemps pris valeur d'icône (et ce, parfois, jusqu'à la trivialisation), il en va sans doute un peu de même pour cet illustrissime cliché daté des alentours de 1898, figurant G. W. "Billy» Bitzer, le futur 
16 Sur cette question, voir donc Wolfgang Schivelbusch, Histoire des voyages en train, op. cit., pp. 39-56 et pp. 57-74 particulièrement. opérateur attitré de Griffith, juché sur le chasse-pierres d'une locomotive américaine, tenant à bout de bras sa lourde caméra Biograph. Autre image emblématique et surinvestie, qui résonne en quelque sorte à l'unisson de la première. Souvent reproduit, ce cliché fameux doit constituer, en un sens, l'une des premières photos de tournage. S'y exhibe en effet, non le produit, mais le dispositif d'une prise de vue. Et frappe en cette image - dont on ne connaît pas grand-chose par ailleurs -, le fait qu'elle construise et énonce tous les termes d'une homologie par dédoublement: sur le plan horizontal, le regard concentré de Bitzer est comme redoublé, symétriquement, par celui du conducteur de la locomotive, dont le visage apparaît à l'arrière-plan, à l'embrasure de la cabine ; mieux encore, sur le plan vertical, l'objectif de l'appareil de prise de vue reproduit comme en réduction, et sur le même axe, le fanal disposé au sommet de la locomotive, juste devant la cheminée. Deux corps et deux regards emportés par le même mobile: tous les termes de l'attelage se trouvent là énoncés. Et, avant même que d'en envisager les produits, il importe de se concentrer sur le mobile seul, en tant qu'il véhicule déjà, en dehors de tout dédoublement, des conditions spécifiques d'exercice du regard.

Ce n'est assurément pas l'un des moindres mérites de l'historien allemand Wolfgang Schivelbusch que d'avoir mis en lumière la profonde crise perceptuelle induite par l'introduction et la généralisation, au cœur du XIX ${ }^{\mathrm{e}}$ siècle, de la locomotion ferroviaire. S'appuyant principalement sur des récits de voyage historiquement distribués, Schivelbusch croit pouvoir y discerner deux conceptions rigoureusement antagoniques, lesquelles renvoient à deux moments spécifiques dans le processus d'intégration dont la nouvelle machine devait faire l'objet 16 .

Suivant la première conception, le voyage ferroviaire s'éprouve dès l'abord dans toute sa force de rupture en regard des données traditionnellement mises en jeu par la locomotion préindustrielle (c'està-dire, pour l'essentiel, le voyage en diligence, produit d'une traction animale). Dans la plupart des récits de voyage de cette première époque, Schivelbusch croit pouvoir déceler la mise en place d'un vaste réseau d'oppositions conventionnelles et systématiques: au trajet labile et serpentant du voyage préindustriel, s'oppose le trajet linéaire et contraint du voyage ferroviaire; à la libre relation entre le véhicule et la voie qui caractérise le voyage en diligence, répond, par contraste, le véritable engrenage du mobile et de sa surface courante propre au voyage en chemin de fer; à la surface accidentée de la route, fait pendant la surface lisse, aplanie (par divers travaux de terrassement - le tunnel, qui 
perce les obstacles - ou de construction - le viaduc, qui les surmonte) de la voie, etc.

L'important, en la circonstance, c'est que la locomotion ferroviaire fut ressentie d'autant plus durement qu'elle faisait fond sur l'abolition de tout substrat matériel et animal. Là où le voyage préindustriel figurait une sorte d'état personnalisé du transport dont tous les paramètres étaient réglés à l'échelle humaine des distances, des rythmes et des durées, là où il autorisait, en fin de compte, une relation harmonieuse et immédiate à la nature (laquelle s'offrait alors comme sollicitation multi-sensorielle, impliquant aussi bien l'ouïe et l'odorat que le goût et le toucher), le voyage industriel, pour sa part, coupe toute relation avec le monde extérieur: tandis que les bruits du dehors sont oblitérés par le vacarme de la machine, le dispositif même du compartiment a pour effet de couper court à toute forme de relations olfactives, gustatives et tactiles avec le paysage traversé. De là que la plupart des premiers commentateurs aient pu s'accorder à dire que le voyage industriel provoquait, par définition, une "perte du paysage", qu'il précipitait un divorce explicite entre le moyen de locomotion et le monde extérieur.

De tous les sens, celui de la vue fut, d'évidence, le plus durement éprouvé - et c'est autour de lui, surtout, que le débat prit ses formes les plus aiguës. Au regard des sujets modelés par les données du voyage préindustriel, la mécanisation du transport introduite par le chemin de fer ne pouvait se vivre, en toute logique, que sur le mode de la perte, de la désorientation, de la dissolution. C'est qu'il y allait fondamentalement de deux logiques des sensations absolument inconciliables: à la série continue d'impressions reçues dans le voyage préindustriel, le voyage ferroviaire substituait une accumulation rapide d'impressions toujours variables et instables. De l'un à l'autre, le renversement est en fait double, qui touche à la fois à un rapport de vitesse et à un rapport de continuité. Dans son principe, le voyage préindustriel instaurait en effet une relation optique relativement stabilisée avec le paysage: développé à vitesse réduite, il autorisait la contemplation d'un monde extérieur perçu comme espace homogène et continu, offert, pour ainsi dire, d'un seul tenant. Or, le propre du voyage mécanisé, ce fut justement de faire éclater pareil mode de perception: mené à toute allure, non seulement interdira-t-il au regard toute attitude contemplative, mais encore lui imposera-t-il d'appréhender le paysage exclusivement sous les espèces d'une démultiplication de données visuelles disjointes, fragmentaires, comme éclatées. Désormais éprouvée sur le mode dysphorique, la nouvelle relation optique au monde extérieur donnera lieu à la construction d'un motif particulièrement récurrent dans les premiers récits de voyage 
17 Sur le Padorama, voir Richard D. Altick, The Shows of London. A Panoramic History of Exhibitions 1600-1862, Cambridge University Press, 1978; et Silvia Bordini, Storia del Panorama: Ia visione totale nella pittura deI XIX secolo, Officina Edizione, Rome, 1984, pp. 284-285.

18 Dès l'année de son inauguration, cette ligne donna d'ailleurs lieu à de nombreux récits, qui, tous, d'une manière ou d'une autre, font état de la désorientation perceptuelle et de la sensation de césure éprouvée entre le monde du compartiment et celui du paysage (à ce sujet, voir notamment le récit anonyme allégué par Wolfgang Schivelbusch, op. cit., pp. 30-31). On sait par ailleurs qu'un dispositif un peu analogue, mais beaucoup plus sophistiqué, fut présenté lors de l'Exposition Universelle de 1900, sous le nom de Panorama Transsibérien: grâce à des toiles mobiles, les spectateurs, installés dans de luxueux wagons appartenant à la Compagnie générale des Wagons-Lits, pouvaient voir défiler sous leurs yeux, à la fenêtre de leur compartiment, les étapes les plus spectaculaires d'un voyage entre Moscou et Pékin. La plupart des ouvrages de référence cités plus haut contiennent des développements consacrés au moving panorama. A ce sujet, voir notamment Stephan Oettermann, Das Panorama. Die Geschichte eines Massenmediums, Syndicat, Frankfurt/Main, 1980, pp. 258-274; Silvia Bordini, op. cit., pp. 277-290 (ch. 17 : "Vedute ottiche et Moving Panoramas") et Ralph Hyde, Panoramania! The Art and Entertrainment of the "All-Embracing" View, Trefoil Publications, Londres, 1988, pp. 131-168 (ch. 6/ "Moving Panoramas"). en chemin de fer: celui, en l'occurrence, de la volatilisation du paysage. Sous l'impulsion de la machine, le paysage devient ainsi la proie d'une dissolution, d'une pulvérisation, d'une dispersion généralisées. Tension optique extrême, mais toujours déçue, vaine, stérile: le voyage ferroviaire n'exaspère ainsi le regard que pour mieux l'aveugler. Rien d'étonnant, dès lors, à ce que certains commentateurs aient cru bon de fustiger les effets profondément abrutissants de l'expérience ferroviaire : la forte sollicitation du regard, conjuguée à la difficulté (voire à l'impossibilité) de rencontrer effectivement le paysage comme visible, finit par basculer en négation et en fatigue (oculaire, physiologique, mentale). Tel est le sens de la fameuse remarque de l'esthéticien anglais John Ruskin, lorsqu'il soutient que "[1] e voyage devient abêtissant à proportion exacte de sa vitesse».

Or, phénomène remarquable - et l'on peut légitimement s'étonner de ce que Schivelbusch ne s'en soit pas avisé -, au moment même où Ruskin et bien d'autres avec lui se dressent avec véhémence contre la "volatilisation du paysage» impliquée par le voyage en chemin de fer, émerge, en Grande-Bretagne précisément, une variante du panorama scénographique, connue sous le nom curieux de Padorama (1834)17. Il s'agit en fait d'un panorama mouvant (moving panorama), articulé autour d'une toile mobile d'environ neuf cents mètres carrés, qui défile devant des spectateurs répartis en plusieurs wagons. Il n'est pas inutile de préciser que cette toile mobile visait à figurer les portions les plus intéressantes, les plus «pittoresques» de la ligne Liverpool-Manchester, ouverte quelques années auparavant et qui fut l'une des plus importantes de son temps 18 .

Dérivé du panorama classique, le moving panorama instaure à son endroit une série de ruptures décisives. Tout d'abord, il brise fondamentalement avec le principe de circularité, pour jouer d'une mise à plat et d'un encadrement de la toile, désormais bornée. Processus d'ouverture, d'aplatissement et de limitation (au sens fort) de l'image qui n'est d'ailleurs pas sans rappeler quelques-uns des principes fondateurs du panorama photographique, et cela même si les deux pratiques et les deux dispositifs se maintiennent par ailleurs en une relation de stricte irréductibilité. D’autre part, prenant son élan sur cette fracture originaire, le moving panorama institue évidemment une mobilisation de la toile: loin que le paysage se donne à voir comme une étendue murale enveloppante, à parcourir selon un balayage visuel de $360^{\circ}$, il s'offre à l'inverse sous les espèces d'un défilement - cette mobilisation de la toile impliquant en retour, conformément à un principe plus haut évoqué, une immobilisation relative du spectateur. S’immobiliser pour mieux saisir 
le déploiement d'une image mobile: voilà qui permettrait de marquer, en amont, une affinité avec le diorama de Daguerre et Bouton et, en aval, bien sûr, de spécifier une parenté avec le dispositif cinématographique, aussi bien dans sa donnée élémentaire que dans certaines de ses actualisations circonstancielles (difficile, évidemment, de ne pas songer au dispositif singulier du Hale's Tour expérimenté aux Etats-Unis à partir de 1904-1905).

C'est peut-être à faire détour par le moving panorama que l'énigme de la vue panoramique est susceptible de connaître un début de résolution. En tout état de cause, les dispositifs de type padoramique apportent un nouvel élément de réponse, partiel mais significatif, à la question initiale. Ce qui ne signifie pas pour autant que la référence au panorama scénographique classique doive être complètement évacuée. Car si le moving panorama s'y oppose en certains points, il ne fait par ailleurs qu'en rejouer assez fidèlement la logique profonde. En somme, ce que le panorama scénographique aura accompli quant à l'espace fuyant, enchevêtré et tentaculaire des grandes villes en pleine expansion, le padorama l'opère pareillement, tout bien pesé, à l'endroit de l'espace disséminé, volatilisé, pulvérisé par la locomotive ferroviaire. De part et d'autre, s'observe ainsi la mise en acte d'une même logique de substitution compensatoire, visant à produire, en dernier ressort, une illusion de puissance et de maîtrise, aussi bien par la restauration d'une visibilité/lisibilité que par une réaffirmation de la pleine souveraineté du regard, celui du sujet urbain comme celui du sujet ferroviaire.

Reconnaître au padorama une fonction d'ordonnancement et de restauration, prendre acte de sa totale solidarité avec l'effet de "volatilisation du paysage» originellement emporté par l'expérience ferroviaire, ne doit pas pour autant occulter l'autre versant de la question. Dans une certaine mesure, en effet, le défilement padoramique ne se sera affirmé dans toute sa nécessité et son efficience qu'à l'endroit de sujets profondément désorientés par leur expérience initiale du voyage ferroviaire, au moment même où ce mode de locomotion est encore ressenti comme étranger et jugé inassimilable à l'aune des données fondatrices du voyage préindustriel. Mais, très vite, parallèlement à la conception conservatrice, émergera une conception progressiste, ouvertement concurrente, qui en renversera un à un tous les postulats. Tout se passe en la circonstance comme si la référence au voyage traditionnel s'estompait progressivement, ouvrant à l'élaboration d'un mode de perception parfaitement accordé à l'expérience ferroviaire. Selon cette conception nouvelle, la vitesse et le trajet en ligne droite de la machine sur une surface plane (ou aplanie) ont pour effet, non de détruire le paysage, de le pulvériser au 
regard, mais bien plutôt d'en permettre, en dernière instance, le complet déploiement, l'accession la plus attrayante au registre du visible. Comme le souligne Schivelbusch:

«Ce qui apparaît alors n'est plus un paysage pittoresque détruit par le chemin de fer, mais c'est inversement un paysage intrinsèquement monotone que le train place dans une perspective esthétique intéressante. Le train met en scène un nouveau paysage. La vitesse, qui, pour la perception d'un Ruskin, volatilise les objets et leur ôte leur existence contemplative, devient pour cette nouvelle perception un élixir de vie. C'est seulement par la vitesse que les objets du monde visible prennent de l'éclat. »19

La divergence ne saurait plus nettement se marquer: d'un côté, la vitesse est pensée comme négation du paysage et comme obstacle à la vision; de l'autre, elle se définit, tout à l'inverse, comme la condition sine qua non de la vision et de l'appréhension du paysage: c'est très précisément parce que le paysage est traversé en trombe qu'il accède à la pleine existence pour le regard et qu'il (re)devient dès lors justiciable d'une expérience esthétique. En somme, c'est le mouvement machinique même qui, au regard du voyageur, définit et désigne le paysage comme paysage.

Dans le même registre, si la notion de vitesse trouve ici à se retourner positivement en une affirmation euphorique, la discontinuité des impressions, leur accumulation, bien loin de figurer un trop de stimulations susceptible d'abrutir le regard - ainsi que Ruskin avait pu le soutenir -, se transforment et s'imposent en l'occurrence comme les données constitutives de l'expérience optique la plus captivante qui soit: la discontinuité se résorbe de la sorte, positivement, en variété, en renouvellement constant des objets et des scènes proposés au regard; le paysage en ressort ainsi toujours neuf, ne cessant de révéler des aspects inédits.

Enfin, la plupart des champions de la conception progressiste n'auront de cesse de souligner le synthétisme de la vision proposé par l'avancée ferroviaire. La vitesse fait si bien apparaître les objets et les scènes dans un rapport de succession immédiat qu'elle a pour effet ultime de réunir, en un même spectacle visuel, ce qui, dans l'expérience $\mathrm{du}$ paysage, demeure effectivement disjoint. C'est alors que le train se consacre en authentique machine optique, engendrant le paysage, le transformant, donnant, sur lui, son point de vue - comme une grande loupe passée sur le monde extérieur, révélant sa topographie, ses creux et ses plis, ses reliefs et ses méandres, etc.

Plus remarquable encore, ce synthétisme de la vision, en vertu duquel la fugacité et la partialité du visible sont précisément tenues, 
l'une et l'autre, pour autoriser la saisie de son tout en une vue d'ensemble (tout le contraire d'une volatilisation, en somme), sollicite pour ainsi dire spontanément, dans le chef de certains observateurs, la métaphore du panorama (on y revient). Tel, par l'exemple, ce texte de Jules Clarétie, publié en 1865 :

"En quelques heures, il [le train] vous présente toute la France, sous vos yeux se déroule la totalité du panorama, une succession rapide d'images charmantes et de surprises toujours nouvelles. Il vous montre purement et simplement l'essentiel d'une région, en vérité c'est un artiste dans le style des anciens maîtres. N'exigez de lui aucun détail mais seulement le tout où est la vie. Enfin, après qu'il vous a ravi par la fougue du coloriste, il s'arrête et vous libère à votre but. » 20

Du mode de vision imposé par le chemin de fer, le panorama (en l'espèce, un panorama mobile, semble-t-il, puisqu'il «se déroule») offrirait donc tout à la fois l'approximation et la référence la plus proche. Traversé par le train, le paysage n'accède pas seulement à l'existence: il trouve à s'engendrer, de surcroît, comme représentation panoramique. Voilà qui livre sans doute une nouvelle clé de l'énigme: le processus qui aura mené de la "volatilisation du paysage» à sa résurrection en panorama consacre, en même temps, l'émergence d'un mode de vision singulier en vertu duquel le regard ne peut dorénavant s'exercer autrement que dans la mobilité. Bien loin de pulvériser le monde extérieur, la mobilité se constitue de la sorte, et au rebours, comme la condition même de l'exercice du regard, comme sa norme fondatrice - et l'on rejoint par ce biais la notion d'" œil variable" avancée par Jacques Aumont 21.

C'est dire, dès lors, que le train n'a évidemment pas attendu le cinéma pour inventer de toutes pièces une vision panoramique : qu'avant même de servir de support à un appareil de prise de vue il aura pu revendiquer pour son propre compte, et de plein droit, le titre d'opérateur. A partir de là, la voie était, si l'on ose dire, grande ouverte pour la vue panoramique de cinéma - aussi bien comme pratique que comme locution.

Une remarque, encore, pour en terminer sur ce point - aussi bien que pour ouvrir une nouvelle piste de recherche, que je me réserve d'explorer en une autre occasion. C'est chose trop peu connue, aujourd'hui, que le terme même de "panorama" se vit peu à peu réapproprié dans le champ des pratiques associées aux projections de lanterne magique. Dans son traité de 1893, Henri Fourtier consacre ainsi un chapitre entier aux "panoramas simples et doubles", qu'il prend soin de qualifier dès l'ouverture :
20 Jules Clarétie, Voyages d'un Parisien, Paris, 1865 , p. 4. Cité dans Wolfgang Schivelbusch, op. cit., pp. 66-67.

21 Jacques Aumont, "L'œil variable, ou la mobilisation du regard", dans L'œil interminable. Cinéma et peinture, Librairie Séguier, Paris, 1989, pp. 37-82. 
22 Henri Fourtier, Les tableaux de projections mouvementés, Gauthier-Villars et Fils, Paris, 1893, p. 27.

23 /d., pp. 33-34
"On donne le nom générique de panoramas à des tableaux allongés qui, en défilant lentement devant un cadre en métal ou en carton noirci, ajouré suivant les dimensions habituelles des caches $(75 \times 75)$ donnent au spectateur l'illusion d'une vue panoramique, c'est-à-dire comprenant une partie du pourtour de l'horizon.»22

Longues vues, en somme, étirées latéralement, et en regard desquelles la totalisation du voir est fonction moins d'une circularité englobante que d'un défilement, d'une translation, c'est-à-dire d'une mobilisation du cadre imparti à la représentation. Proches, en leur principe, du moving panorama, ces vues panoramiques s'en démarquent néanmoins en cela qu'au dispositif de la toile (mobile), elles substituent une simple plaque allongée, requérant, selon les cas et les effets recherchés, une lanterne simple ou double. C'est sans doute par cette voie que la logique panoramique fit son entrée dans la tradition des pratiques de projection (cette tradition même qui allait peser de tout son poids sur la composition conune sur l'exhibition des premières vues animées). Quant aux motifs privilégiés par ces plaques à coulisse (que Fourtier nomme également «tableaux à longue course», eu égard au déplacement imprimé au tiroir), ils furent très divers: mais l'on sait que les panoramas maritimes et ferroviaires formaient l'essentiel de cette production, ainsi que Fourtier y insiste d'ailleurs à la clôture de son exposé:

"On obtiendra un silhouettage exact du premier tableau, qui constituera la cache du second tableau: derrière cette cache, on ferra glisser le panorama des rives du fleuve ou de la mer, sur lequel la première scène a été prise, et la projection donnera au spectateur l'illusion complète du mouvement de translation du passager, par suite de cet effet spécial qui nous fait croire que nous sommes en mouvement quand les lignes d'horizon se mettent à fuir devant nous, effet que chacun a pu observer dans une gare, lorsqu'étant soi-même dans un wagon immobile, on voit s'ébranler un train voisin. L'exemple que nous venons de citer suffira pour indiquer au lecteur le mode opératoire. Nous signalerons comme sujets du même genre faciles à exécuter: des voyageurs en wagon voyant fuir le paysage à travers les portières, des matelots regardant par un sabord ouvert l'arrivée au port, etc.» $\mathbf{2 3}$

Il va sans dire que seule la dimension de la latéralité s'offrait à ce type de translation.

De la gondole au train en mouvement, le glissement n'aura été, somme toute, que très logique. Comme l'on sait, le panorama ferroviaire s'imposera rapidement comme un genre à part entière, dont la grande vogue 
s'étalera approximativement sur une dizaine d'années, de 1896 à 1906. Toute la relation nouée entre le train et le cinéma trouvera là une autre manifestation exemplaire, car toute littérale, métonymique plutôt que métaphorique: cinéma sur train, les deux machines accouplées, emportées dans le même mouvement de traversée.

C'est en ce genre singulier que le premier cinéma trouvera à s'énoncer très littéralement sur le dos du train ("cinéma à vapeur», comme le dira pertinemment André Labarthe à propos de Lumière). Car si le train invente déjà, à lui seul, un mode de vision panoramique, l'appareil de prise de vue qui vient s'arrimer à lui dès 1896 se bornera en somme à rendre compte de cette vision. Son office ne sera donc pas de production, mais de simple reproduction d'une image toujours déjà là, qui, en quelque sorte, lui préexistait. Du phénomène de transformation du paysage en spectacle "panoramique", le panorama ferroviaire offre ainsi quelque chose comme l'aboutissement logique. En son sein, le paysage n'existe plus, et cette fois à la lettre, qu'au titre d'image. Image d'image, la vue panoramique s'articule donc également au principe d'une médiation de médiation: à cet égard, elle doit être tenue pour une représentation seconde, lors même que cette secondarité ne s'y trouverait pas physiquement et explicitement marquée.

Cet aspect de la vue panoramique n'est évidemment pas pour rien dans son pouvoir de fascination - et l'on aura à s'interroger sur les effets qu'elle entend cultiver de ce point de vue. Pour l'heure, il conviendra d'abord de se demander en quoi le panorama ferroviaire représente effectivement une nouvelle élaboration de ce qui nous regarde au sein de ce que nous voyons; en quoi se spécifie, dans son sillage, une nouvelle variété d'image-attraction, comme retournement et complément logique de la vue attentatoire.

Afin de se placer au plus près de ce retournement fondamental, il importe de prendre acte de la relative polysémie enfermée dans le terme générique de "panorama» ou de "vue panoramique». Sous une unité de principe (l'attelage des machines), la pratique du panorama cinématographique dissimule en effet une grande diversité de postures de tournage - comme si, très tôt, il s'était agi, pour des motifs divers, d'explorer le train sous toutes ses facettes, dans tous ses sites possibles, et de réinventer constamment de nouvelles modalités d'attelage (ce jeu réglé d'essais et de variations fixant d'ailleurs tous les termes d'un véritable expérimentalisme primitif: on essaie, puis on voit ce que ça donne). 